\title{
ALGEBRAS WITH ACTIONS AND AUTOMATA
}

\author{
W. KUHNEL and $M$. PFENDER \\ Fachbereich Mathematik \\ TU Berlin, Str.d. 17. Juni 135 \\ 1000 Berlin 12, Germany

\section{J. MESEGUER and I. SOLS} \\ Mathematics Department \\ University of California \\ Berkeley, California 94720 U.S.A. \\ (Received February 18, 1980)
}

\begin{abstract}
In the present paper we want to give a common structure theory of left action, group operations, R-modules and automata of different types defined over various kinds of carrier objects: sets, graphs, presheaves, sheaves, topological spaces (in particular: compactly generated Hausdorff spaces). The first section gives an axiomatic approach to algebraic structures relative to a base category B, slightly more powerful than that of monadic (tripleable) functors. In section 2 we generalize Lawveres functorial semantics to many-sorted algebras over cartesian closed categories. In section 3 we treat the structures mentioned in the beginning as many-sorted algebras with fixed "scalar" or "input" object and show that they still have an algebraic (or monadic) forgetful functor (theorem 3.3) and hence the general theory of algebraic structures applies. These structures were usually treated as one-sorted in the Lawvere-setting, the action being expressed by a family of unary operations indexed over the scalars. But this approach cannot, as the one developed here, describe continuity of the action (more
\end{abstract}


general: the action to be a B-morphism), which is essential for the structures mentioned above, e.g. modules for a sheaf of rings or topological automata. Finally we discuss consequences of theorem 3.3 for the structure theory of various types of automata. The particular case of algebras with fixed "natural numbers object" has been studied by the authors in [23].

KEY WORDS AND PHRASES. Algebras, actions, automata, algebraic functor.

1980 MATHEMATICS SUBJECT CLASSIFICATION CODES. 08A05, 18C15, 68D05.

\section{INTRODUCTION (ALGEBRAIC FUNCTORS)}

The following paper is directed to specialists. Customary shorthand theorems, and arguments are freely used. Triple theory (cf. [1], [2]VI, [3]3) showed that the notion of "algebraic structures" should be viewed as a relative notion: structures of a given species are algebraic over some other category, and this is best described in terms of the forgetful functor $\mathrm{U}: \underline{\mathrm{K}} \rightarrow \underline{B}$ from some category $\underline{\mathrm{K}}$ of "algebras" into a "base" category B. We say U is tripleable or monadic (cf. references above). We will use only the axiomatic properties of monadic functors. 1.1 PARÉ-CRITERION (cf. [2]VI.7) U has a left adjoint F, and U creates absolute coequalizers, i.e. coequalizers preserved by any functor.

We will use as our basic notion the following slightly stronger one: 1.2 Definition AND CHARACTERIzation. A functor $\mathrm{U}: \underline{\mathrm{K}} \rightarrow \underline{B}$ is algebraic, if

(i) U has a left adjoint F

(ii) U creates (inverse) Iimits

(iii) U creates coequalizers of U-kernel pairs (K-pairs mapped by U into kernel pairs), i.e. quotients by congruences can be 
calculated downstairs.

For $\underline{B}$ finitely complete, algebraic functors are just monadic ones which satisfy (iii). This follows from the characterization of monadic functors in [4]3.3.

$\mathrm{U}$ is of finite rank if (iv) U creates filtered colimits.

1. 3 REMARK. For $\underline{B}=$ Sets, "monadic" and "algebraic" are the same, again by [4], 3.3, since in sets coequalizers are retractions. As we will see in section 2, "usual" algebraic structures, i.e. those with operations of finite arity (in particular L a w v r e - type algebras) have algebraic forgetful functors of finite rank, if $\underline{B}$ has enough properties of sets. We prefer "algebraic" to "monadic" because of the following quite important structure theorems and because generation of congruences, isomorphism theorems, $\mathrm{Z}$ a $\mathrm{s}$ e $\mathrm{n}$ h a $\mathrm{u}$ lemma, $\mathrm{J}$ o $\mathrm{d}$ a $\mathrm{n}$ $H \ddot{H} l \mathrm{~d}$ e $\mathrm{r}$ theorem hold for $\underline{\mathrm{K}}$ with algebraic $\mathrm{U}: \underline{\mathrm{K}} \rightarrow \underline{\mathrm{B}}$ into $\underline{B}$ complete we11-powered and cocomplete, see [5], [6].

1. 4 LIFTING THEOREM. Let $U: \underline{K} \rightarrow \underline{B}$ be algebraic. If $\underline{B}$ is complete, we11-powered, and cocomplete, then so is $\underline{K}$. If $\underline{B}$ has kernel pairs and a coequalizer-mono factorization, then so has $\underline{K}$. PROOF. Since U creates limits, completeness of $\underline{B}$ implies completeness of $\underline{\mathrm{K}}$. For a $\underline{B}$-monomorphism $B \stackrel{\mathrm{m}}{\longrightarrow}$ UK there is at most one $\underline{K}-$ (mono)morphism $\mathrm{K}^{\prime} \stackrel{\mathrm{m}^{\prime}}{\longrightarrow} \mathrm{K}$ such that $\mathrm{Um}^{\prime}=\mathrm{m}$ : If such an $\mathrm{m}^{\prime}$ exists, then the left column in
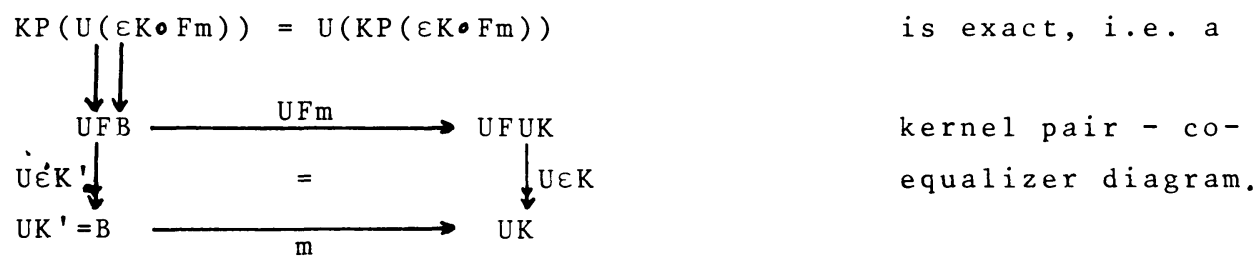

UEK' is uniquely determined by $B$ since $m$ is mono, and, being a retraction, UEK' is a coequalizer of its kernel pair.since U creates 
coequalizers of U-kernel pairs, especially of $K P(\varepsilon K \bullet F m)$, there is a unique $F B \longrightarrow K^{\prime}$ mapped by $U$ into $U F B \stackrel{U \varepsilon K^{\prime}}{\longrightarrow} B$, namely $\mathrm{FB} \stackrel{\varepsilon K^{\prime}}{\longrightarrow} \mathrm{K}^{\prime}$. So m admits at most one $\mathrm{K}^{\prime} \stackrel{\mathrm{m}^{\prime}}{\longrightarrow} \mathrm{K}$ with Um' $=\mathrm{m}$, hence $\underline{B}$ well-powered implies $\underline{K}$ well powered. Cocompleteness: Since U creates coequalizers of (U-)kernel pairs, and $\underline{B}$ has coequalizers of kernel pairs, $\underline{K}$ has them too. A general theorem (cf. [7] 3.53) then gives coequalizers of arbitrary pairs $A \underset{g}{\stackrel{f}{\longrightarrow}} K$ in $K$ : take the coequalizer of the intersection over the set ( $\underline{K}$ well-powered) of all kernel pairs on $K$ through which $\underset{\mathrm{g}}{\stackrel{\mathrm{f}}{\rightleftarrows}}$ factors, this intersection being a kernel pair. Coproducts in $\underline{K}$ may now be constructed as follows (cf. the wellknown "free product"-construction for groups):

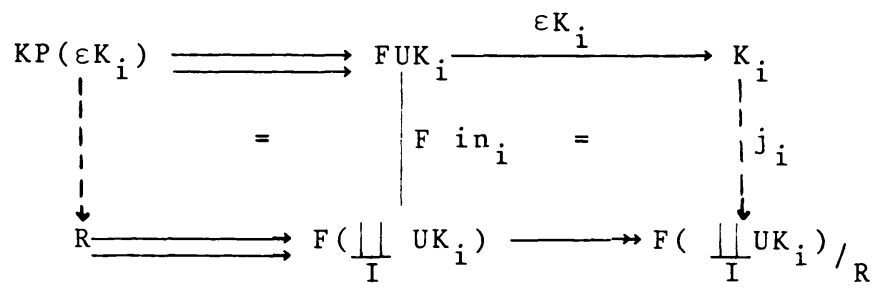

$R$ is the kernel pair generated by the pairs $\mathrm{KP}\left(\varepsilon \mathrm{K}_{\mathrm{i}}\right) \Longrightarrow \stackrel{\mathrm{Fin}}{\longrightarrow} \stackrel{\mathrm{i}}{\longrightarrow}$. By construction of $R$, there is a unique $j_{i}$ making the right hand

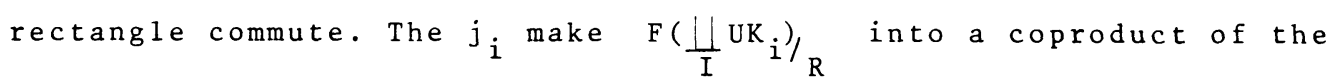
$\mathrm{K}_{\mathrm{i}}$, verification being a simple diagram chase. This shows that $\underline{K}$ is cocomplete. By replacing $\Perp$ by other colimits, the same arguments show: For $\underline{B}$ complete and wel1-powered, $\underline{K}$ is cocomplete at least as far as $\underline{B}$ is.

Factorization: For $\mathrm{K} \stackrel{\mathrm{f}}{\longrightarrow} \mathrm{K}^{\prime}$ let $\mathrm{UK} \stackrel{\mathrm{q}}{\longrightarrow} \mathrm{D} \stackrel{\mathrm{m}}{\longrightarrow} \mathrm{UK}$ be the coequalizer-mono factorization of Uf. $q=\operatorname{coequ}(K P(U f))=\operatorname{coequ}(U(K P f))$, since $U$ creates kernel pairs. By U algebraic, there exists a coequalizer $\mathrm{K} \stackrel{\tilde{\mathrm{q}}}{\longrightarrow} \tilde{\mathrm{C}}$ of $\mathrm{KPf}$ over $\mathrm{q}$ and hence a factorization $\mathrm{K} \stackrel{\tilde{q}}{\longrightarrow} \mathrm{C} \stackrel{\tilde{m}}{\longrightarrow} \mathrm{K}$ over that of Uf. $\tilde{\mathrm{m}}$ is mono, 
since monos are characterized by the pair of identities to be kernel pair, and U creates kernel pairs. q.e.d.

There is no assumption on $\underline{K}$ in the theorem. $L$ i $n$ o $n$ shows in [8], corollary 2 cocompleteness of $\underline{K}$, if $U$ is monadic and $\underline{K}$ already has coequalizers of reflexive pairs.

1.5 PRESENTATION OF ALGEBRAS. For $\underline{B}$ complete and wellpowered with coequalizers, $U: \underline{K} \longrightarrow \underline{B}$ algebraic, any $B \in \underline{B}$ ("set" of generators) together with $\frac{\mathrm{P}_{1}}{\mathrm{P}_{2}} \mathrm{UFB}$ ("relations") presents a K-object, i.e. the coequalizer FB/G of $\mathrm{p}_{1}, \mathrm{p}_{2}$ (in $\underline{K}$ relative U, cf. [8]). Each $K \in \underline{K}$ has a presentation, namely $\mathrm{UKP}(\varepsilon \mathrm{K}) \Longrightarrow \mathrm{FUK} \stackrel{\varepsilon \mathrm{K}}{\longrightarrow} \mathrm{K}$.

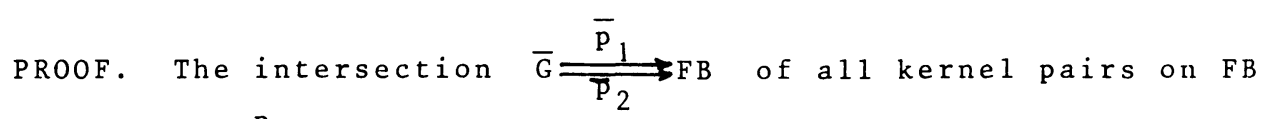
through which $\underset{\mathrm{p}_{2}}{\stackrel{\mathrm{p}_{1}}{\longrightarrow}} \mathrm{UFB}$ factors (in $\underline{B}$ ) exists (ㅡㅡ well-powered and complete by 1.4 ) and is a (U-)kernel pair [7], 3.51). Hence, by U monadic, $F B \longrightarrow F B / \bar{G}=: F B / G$ ex: ats and has the desired property. Second part of the assertion: $U \varepsilon K$ is a retraction (for $\eta U K$ ), hence coequalizer of its kernel pair UKP( $\varepsilon K)$, so $\varepsilon K$ is a coequalizer relative $U$ of $\operatorname{UKP}(\varepsilon K)$, bfcause $U$ is faithful. q.e.d.

1.6 COMPOSITION THEOREM. If in $\underline{\mathrm{K}} \stackrel{\mathrm{U}}{\longrightarrow} \underline{\mathrm{B}} \stackrel{\mathrm{V}}{\longrightarrow} \underline{\mathrm{C}}$, U and $\mathrm{V}$ are algebraic [of finite rank] then so is their composition V U.

PROOF. VU is right adjoint as composition of right adjoints, and creates limits, because $U$ and $V$ do (create them in two steps). Now let $\mathrm{R} \underset{\mathrm{P}}{\stackrel{\mathrm{P}_{\mathrm{O}}}{\mathrm{P}}} \mathrm{K}$ be a VU-kernel pair and VUK $\stackrel{\mathrm{q}}{\longrightarrow} \mathrm{C}=\operatorname{coequ}\left(\mathrm{VUp}_{\mathrm{O}}, \mathrm{VUp}_{1}\right) \cdot\left(\mathrm{Up}_{\mathrm{O}}, \mathrm{Up}_{1}\right)$ being a V-kernel pair (trivial), V algebraic implies unique existence of UK $\tilde{q} \longrightarrow \tilde{C}$ in $B$ with $V \tilde{q}=q$, and $\tilde{q}=\operatorname{coequ}\left(U p_{0}, U p_{1}\right)$. Since $V$ creates 1 imits, 
especially kernel pairs, $\left(U_{p_{0}}, U p_{1}\right)$ is a kernel pair in $\underline{B}$ of $\tilde{q}$, so $\left(p_{0}, p_{1}\right)$ is a U-kernel pair. By U algebraic, there exists a unique $K \stackrel{\tilde{q}}{\longrightarrow} \tilde{\tilde{C}}$ in $\underline{K}$ such that $U \tilde{q}=\tilde{q}$ (hence $\operatorname{vU\tilde {q}}=q$ ), and $\tilde{\tilde{q}}=\operatorname{coequ}\left(\mathrm{p}_{0}, \mathrm{p}_{1}\right)$. Uniqueness of $\tilde{\tilde{q}}$ with respect to $\mathrm{vU} \tilde{\tilde{q}}=\mathrm{q}$ is verified stepwise. If $U$ and $V$ create filtered colimits, then VU does: create them in two steps. So VU is of finite rank. q.e.d. Composition of monadic functors is not monadic in general. The forgetful functors $\mathrm{Cat} \longrightarrow$ Graph and Graph $\rightarrow$ Sets $^{2}$ are monadic, their composition is not, since images of functors need not to be ( sub) categories.

1.7. TRIANGLE THEOREM. If $\underline{\mathrm{K}} \stackrel{\mathrm{U}}{\longrightarrow} \underline{\mathrm{B}} \stackrel{\mathrm{V}}{\longrightarrow} \underline{\mathrm{C}}, \underline{\mathrm{K}}$ has coequalizers, and $W=V U$, then if $V$ and $W$ are monadic, so is $U$.

If $\underline{C}$ is complete, cocomplete and well-powered and if $V$ and $W$ are algebraic [of finite rank], then so is U.

PROOF. U right adjoint follows from [9] theorem 1 (adjoint triangles):

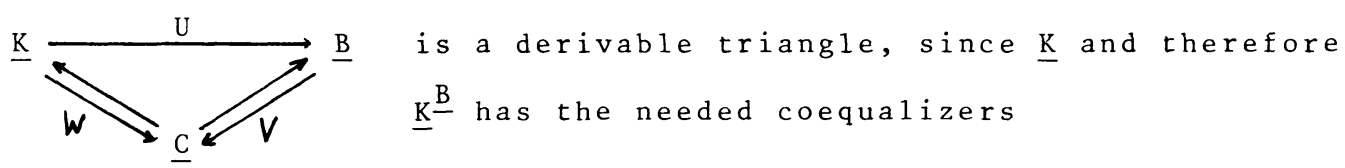
and $\quad \mathrm{FV} \stackrel{\varepsilon}{\longrightarrow} \mathrm{id}_{\underline{B}}=\operatorname{coequ}(\mathrm{FVFV} \stackrel{\mathrm{FV} \varepsilon}{\stackrel{\mathrm{FVV}}{\longrightarrow}} \mathrm{FV})$

by $\mathrm{V}$ being monadic.

By $P$ a $r$ '́'s monadicity criterion we need to show (for the first part of our assertion) that U creates absolute coequalizers.

But since $V$ preserves absolute coequalizers (as such, any functor does), U creates them.

Second assertion: It is easy to verify that a functor (e.g. V,W) into a category having limits, colimits of a given kind which creates these limits, colimits respectively, preserves them. This proves the second part of the assertion: U creates "things" because 
$V$ preserves them, $W$ creates them, and $U$ maps them into the original things, because $V$ creates (uniqueness). U right adjoint holds by the first assertion since the lifting theorem provides a 11 coequalizers in $\underline{K}$ via W. q.e.d.

Each time a (full) subcategory is equationally defined, we will use the following theorem for proving the inclusion functor to be a 1 gebraic.

\subsection{BIRKHOFF-INCLUSION THEOREM. For complete, wel1-} powered $\underline{K}$ having coequalizers and a coequalizer-mono factorization, a full inclusion $I: \underline{L} \longleftrightarrow \underline{K}$ is reflective with coequalizers as reflections (regular epi-reflective), if $\underline{L}$ is closed under products and (mono-)subobjects. It is algebraic, if, in addition, $\underline{L}$ is closed under coequalizers of kernel pairs, i.e. it is a B irkhof f - subcategory.

PROOF. Apply the non-numerated "Satz" on page 96 of [10] taking $M=$ class of all $\underline{K}$-monos, $\Sigma=$ class of all regular epis (coequalizers). $\underline{K}$ is E-cowellpowered, since it is well-powered (bijective correspondence between kernel pairs and (regular) quotients) and satisfies the diagonal-condition for $E, M$ since every regular epi is coequalizer of its kernel pair.

Explicit construc.

Given $K \in \underline{K}$, take out of a representative family (one representant for each iso-class) of regular quotients the family $\mathrm{K} \stackrel{f}{\longrightarrow} \mathrm{L}_{i}$ of those quotients $\backslash \quad\left\langle L_{i} \in \underline{L}\right.$. Then the regular epi-monofacto-

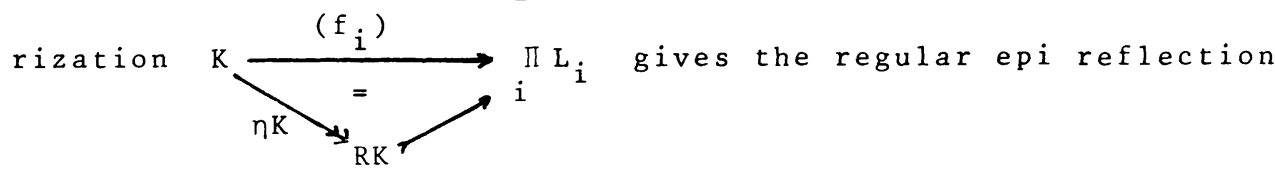

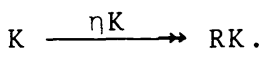

The second assertion is obvious by definition of "algebraic". 


\section{MANY - SORTED ALGEBRAS}

This section generalizes two principal results of [11] to manysorted (heterogeneous) algebras over cartesian closed categories: "theories are algebras" (theorem 2.3) and "models of algebraic theories have algebraic forgetful functors of finite rank" (theorem 2.6).

The case of Sets as base category has been treated in [12], [13], and [14]. A further generalization to the case of (closed) monoidal categories is given in [15] (one-sorted algebras) and in the unpublished [16] (many-sorted algebras).

2.1 DEFINITIONS: For a set I, $\Sigma \in$ Sets $^{I^{*} \times I}$ is called an I-sorted algebraic type or scheme of operators (cf. [12], [13]). $\Sigma$ may be seen as a directed graph with node set I*; $\Sigma \in G r a p h I^{*}$, which has only arrows with codomain in I (coarity 1 ).

A $\Sigma$-model in a category $\underline{B}$ with 1 and given binary product $x$ is a graph-morphism M: $\sum \longrightarrow \underline{B}$ which preserves products: $I^{*} \ni A=i_{1} \ldots i_{n} \longmapsto M A=M i_{1} \times \ldots \times M i_{n}=\left(\ldots\left(M i_{1} \times M i_{2}\right) \times \ldots\right) \times M i_{n}$. I* $31 \longmapsto 1$.

Example: $I=\{\mathrm{R}, \mathrm{A}\}, \quad \Sigma$ :

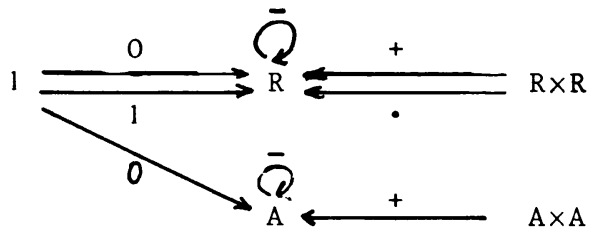

(where we wrote $R \times A$ instead of $R A \in I^{*}$ ) $R \times A$

is the scheme of left modules (over varying rings). Modules are $\Sigma$-models satisfying the usual equations. 
A $\underline{\Sigma \text {-homomorphism }} \mathrm{f}: \mathrm{M} \longrightarrow \mathrm{M}^{\prime}$ is a family

$$
f=(f i)_{i \in I} \in \operatorname{sets}^{I}\left((M i),\left(M^{\prime} i\right)\right)
$$

compatible with the operations. For each $A=i_{l} \ldots i_{n} \stackrel{w}{\longrightarrow}$ i in $\Sigma$

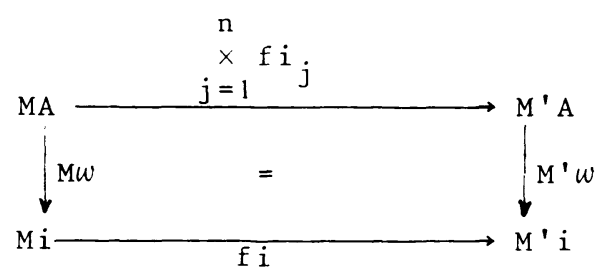

This defines a category $\operatorname{Mod}(\Sigma, \underline{B})$ with forgetful functor $\mathrm{U}: \operatorname{Mod}(\Sigma, \underline{\mathrm{B}}) \longrightarrow \underline{\mathrm{B}}^{\mathrm{I}}, \mathrm{M} \longrightarrow(\mathrm{Mi})_{\mathrm{i} \in \mathrm{I}}$.

\subsection{THEOREM :}

(i) $U: \operatorname{Mod}(\Sigma, \underline{B}) \longrightarrow \underline{B}^{I}$ has a left adjoint, if $\underline{B}$ has and if $B \times-$ preserves coproducts (and hence $x$ distributes over coproducts)

(ii) $U: \operatorname{Mod}(\Sigma, \underline{B}) \longrightarrow \underline{B}^{I}$ creates 1 imits ( $\underline{B}$ arbitrary)

(iii) U creates absolute coequalizers (

(iv) U creates coequalizers of U-kernel pairs, if _ $x_{-}$simultaneously preserves coequalizers of kernel pairs, in particular if $\underline{B}$ is cartesian closed and regular epis (coequalizers) are closed under composition.

(v) U creates filtered colimits, if $B \times-$ preserves them (in particular if $\underline{B}$ is cartesian closed).

The proof is a purely formal extension of that of theorem 1.4 in [15]. For $\underline{B}=$ Sets, (i) and (iv) are proved in [12] and [13].

PROOF. For $\underline{B}=$ Sets, [12] and [13] prove (i) by construction of (free) word algebras over given (Bi) Esets ${ }^{I}$. By analysis of the structure of these word algebras the following proof in the general case is suggested. 


\section{Clone of (composite) operations of $\Sigma:$}

Define $\bar{\Sigma} \in \operatorname{Sets}^{I^{*} \times I^{i}} \subset \operatorname{Graph}_{I^{*}}$ recursively as follows:

arrows $i \stackrel{i d}{\stackrel{i}{\longrightarrow}} i$ are in $\bar{\Sigma} \quad(i \in I)$.

For $\quad A=i_{1} \ldots i_{n} \stackrel{w}{\longrightarrow} i$ in $\Sigma$ and $A_{j} \stackrel{w_{j}}{\longrightarrow} i_{j}$ in $\bar{\Sigma}$

$(1 \leq j \leq n), \quad \underset{j \leq n}{x A_{j}}=A_{1} \ldots A_{j} \stackrel{w\left(w_{1}, \ldots, w_{n}\right)}{\longrightarrow}$ is in $\bar{\Sigma}$

(and that is a 11 ).

For each $A \in \operatorname{Mod}(\Sigma, \underline{B})$ there is a unique extension $\bar{A}: \bar{\Sigma} \longrightarrow \underline{B}$ compatible with substitution, namely $\bar{A} i_{i}=i_{B i}$, $\bar{A} w\left(w_{j}\right)=A \omega \bullet \underset{j \leq n}{\times} \bar{A}_{j}$, and each $f: A \longrightarrow A^{\prime}$ in $\operatorname{Mod}(\Sigma, \underline{B})$ is compatible also with all composite operations w in $\bar{\Sigma}$ (proof by induction).

Construction of $F B$ for $B=(B i)_{i \in I} \in \underline{B}^{I}$.

Abbreviations: $\operatorname{dom}(A \stackrel{\mathrm{W}}{\longrightarrow} \mathrm{i}):=\mathrm{A}($ (domain"),

$\operatorname{cod}(\mathrm{A} \stackrel{\mathrm{w}}{\longrightarrow} \mathrm{i}):=\mathrm{i}\left(\right.$ "codomain"), $\bar{\Sigma}_{i}:=\{w \in \bar{\Sigma}: \operatorname{cod} w=i\}$, $B_{A}=B i_{1} \ldots i_{n}:=\underset{j \leq n}{\times} B i_{j}$.

Then define the i-th carrier object FBi of $F B$ by

$\mathrm{FBi}={\frac{\|}{w \in \Sigma_{i}}}_{B_{\text {dom }} w}, \quad$ injections $B_{\text {dom } w} \stackrel{\text { in } w}{\longrightarrow} F B i$.

How to define $F B\left(A=i_{1} \ldots i_{n}\right) \stackrel{w}{\longrightarrow}$ i) ?

Consider the following àiagram:

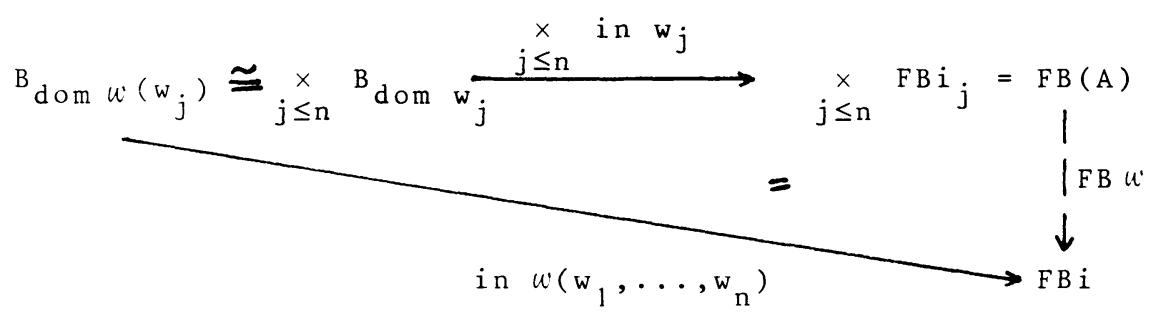

By distributivity of $x$ with respect to coproducts, the rows above 
$\left(\left(w_{j}\right) \in \underset{j \leq n}{\times} \bar{\Sigma}_{i j}\right.$ constitute a coproduct, hence the in $w\left(w_{j}\right)$

induce a morphism $F B w$. This clearly defines $F B \in \operatorname{Mod}(\Sigma, \underline{B})$.

Front adjunction $\eta B=\left(\text { in } i_{i}\right)_{i \in I}:(B i) \longrightarrow(F B i)$.

Universal property of $\eta B:(B i) \longrightarrow F B:$

For a homomorphic extension $\overline{\mathrm{f}}: \mathrm{FB} \longrightarrow \mathrm{A}$ of $\mathrm{f}: \mathrm{B} \longrightarrow$ UA, $i_{1} \ldots i_{n} \stackrel{w}{\longrightarrow}$ in $\bar{\Sigma}$ consider the diagram

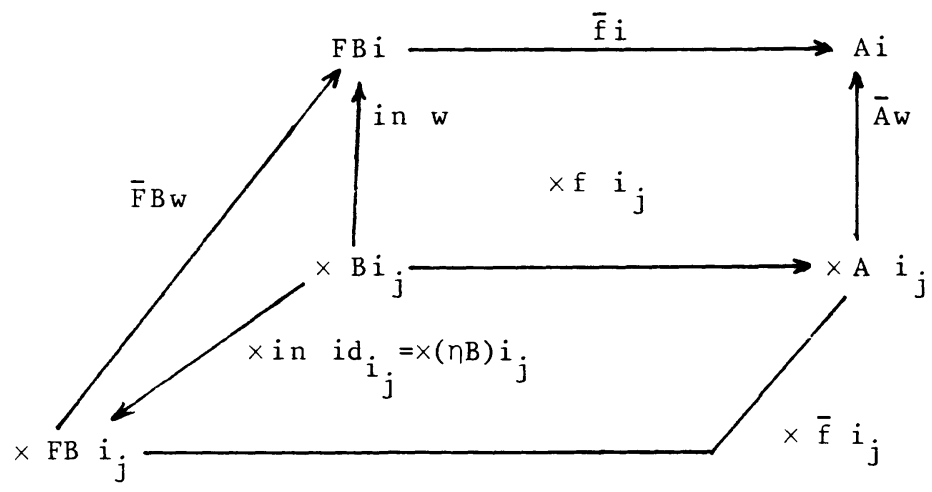

The lower triangle commutes, since $\bar{f}$ extends $f$; the frame commutes, since $\bar{f}$ is compatible with any $w: i_{1} \ldots i_{n} \longrightarrow i$ in $\bar{\Sigma}$.

An induction on $\bar{\Sigma}$ shows the left triangle to commute. Hence $\overline{\mathrm{f}}$ i is necessarily induced out of the coproduct by the $\bar{A} w \circ \underset{j \leq n}{f_{j}}$.

This $\bar{f}$ extends $f\left(t a k e ~ w=i d_{i}\right)$ and is a $\Sigma$-homomorphism as is shown by using the "coproduct-row" and commutativity of (1), the commutative upper rectangle in (2), and the recursive definition of $\bar{A}$. This proves (i).

(ii) is proved straight forward by using the universal property of Lim in the $i-t h$ component.

For (iii) and (iv), consider a pair $R \underset{g}{\stackrel{f}{\Rightarrow}}$ in $\operatorname{Mod}(\Sigma, \underline{B}$ ) which gives rise to an absolute coequalizer diagram or a kernel pair coequalizer diagram $U R \stackrel{\mathrm{Uf}}{\mathrm{Ug}} \mathrm{UM} \stackrel{\mathrm{q} \longrightarrow \mathrm{C}}{\longrightarrow}$ in $\underline{B}^{\mathrm{I}}$ respectively (hence - case (iv) $-\mathrm{Ri} \stackrel{\mathrm{fi}}{\mathrm{gi}} \mathrm{Mi} \stackrel{\mathrm{gi}}{\longrightarrow} \mathrm{Ci}$ is such a diagram). 
Then, by hypothesis, for each $A=i_{1} \ldots i_{n} \stackrel{\omega}{\rightarrow} i$ in $\Sigma$, the upper row in

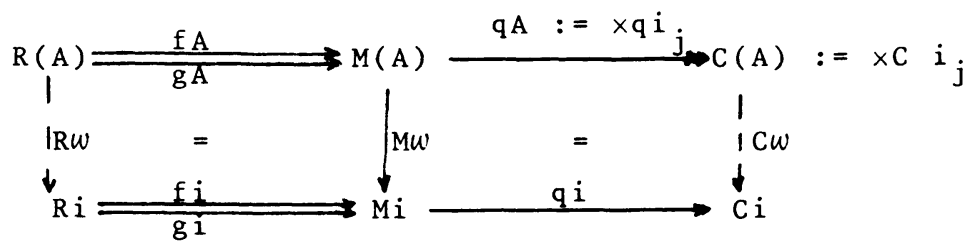

is a coequalizer diagram, hence induces a unique cw compatible with q. So there exists a unique $\tilde{C} \in \operatorname{Mod}(\Sigma, \underline{B})$ over $C$ such that $\mathrm{q}: \mathrm{M} \longrightarrow \tilde{\mathrm{C}}$ is a homomorphism. A simple diagram chase shows q to be a coequalizer of $f$ and $g$ in $\operatorname{Mod}(\Sigma, \underline{B})$.

(v) is proved similarly using the fact that a binary functor $x$ which preserves filtered colimits in each place, commutes with them, see $[5], 6.5$.

Let us now turn to many-sorted algebraic theories and their models.

\subsection{DEFINITIONS: An I-sorted algebraic theory is a category}

$\mathrm{T}$ with object set $|\mathrm{T}|=I^{*}$ (free monoid over I), given products $\left[A=i_{1} \ldots i_{n} \stackrel{P_{j}}{\longrightarrow} i_{j}\right]_{j=1}, \ldots, n, i_{j} \in I$, and $1 \in I$ terminal.

A morphism $t: T \longrightarrow T^{\prime}$ of such theories is a functor which is identity on objects and preserves the given projections. This defines a category Th ${ }_{I}$ with obvious forgetful functor $\mathrm{V}: \mathrm{Th} \mathrm{I} \mathrm{Graph}_{\mathrm{I}^{*}}$. For $\underline{B}$ with given finite product, a $\underline{\mathrm{T}-\mathrm{model}}$ in $\underline{B}$ is a functor $M: T \longrightarrow \underline{B}$ which preserves the given finite products. A T-homomorphism $\mathrm{f}: \mathrm{M} \longrightarrow \mathrm{M}^{\prime}$ is a transformation compatible with $x$, i.e. a family $f=(f i)_{I} \in \underline{B}^{I}((M i)$, (Mi)) satisfying (1) in 2.1 for all $\mathrm{A} \stackrel{\omega}{\longrightarrow}$ i in $T$. This defines the category Funct $(T, \underline{B})$ of T-models in $\underline{B}$ with forgetful functor $U:$ Funct $(T, \underline{B}) \longrightarrow \underline{B}^{I}$.

2. 4 THEOREM: The forgetful functor $\mathrm{V}: \mathrm{Th}_{\mathrm{I}} \longrightarrow \mathrm{Graph} \mathrm{I}^{*}$ Sets $\mathrm{I}^{*} \times \mathrm{I}^{*}$ is algebraic of finite rank (cf.1.2). 
PROOF: I-sorted theories are $\ominus$-algebras in sets for the following $I^{*} \times I^{*}-$ sorted scheme of operators:

$\theta: 1 \stackrel{\text { id }}{\longrightarrow}(A, A), \quad A \in I^{*}$ ("identities")

$(A, B)(B, C) \stackrel{0}{\longrightarrow}(A, C), \quad A, B, C \in I^{*}$ ("composition")

$\left[1 \stackrel{p{ }_{j}}{\longrightarrow}\left(i_{1} \ldots i_{n}, i_{j}\right)\right]_{j=1}, \ldots, n, n \in \mathbb{N}, i_{,} i_{j} \in I \quad$ ("projections")

$\left(A, i_{1}\right) \ldots\left(A, i_{n}\right) \underset{(\bullet, \ldots, \bullet)}{\longrightarrow}\left(A, i_{1} \ldots i_{n}\right), n \in \mathbb{N}, A \in I^{*}, i_{j} \in I$

("induced arrows").

$T \in \operatorname{Mod}(\theta$, Sets) is a theory, iff the following hold:

neutrality of id, associativity of $o, p_{j}\left(f_{1}, \ldots, f_{n}\right)=f_{j}$

(defining equation for induced arrows), $\left(p r_{1} f, \ldots, p r f\right)=f$

for $f: A \longrightarrow i_{1} \ldots i_{n}$ (uniqueness of the induced arrow); cf. [24].

By theorem 2.2, $\operatorname{Mod}(\Theta, \operatorname{Sets}) \longrightarrow$ Sets $I^{*} \times I^{*}$ is algebraic of finite rank Th $I \subset \operatorname{Mod}(\theta$, Sets $)$ is a full subcategory defined by equations, hence (easy verification) closed under subobjects, products, quotients, and filtered colimits. By lifting and $B$ i $r$ h o f inclusion theorem, Th $\longrightarrow$ I Mod $(\Theta$, Sets) is algebraic of finite rank, hence by the composition theorem also $\mathrm{Th}_{\mathrm{I}} \stackrel{\mathrm{V}}{\longrightarrow} \mathrm{Graph}_{\mathrm{I}}=\mathrm{Th}_{\mathrm{I}} \longrightarrow \operatorname{Mod}(\Theta, \operatorname{sets}) \longrightarrow \operatorname{sets}^{*} \times \mathrm{I}^{*} \stackrel{\sim}{=} \mathrm{Graph}_{\mathrm{I}^{*}}$. We draw now some conclusions from this theorem which will be needed for the proof of the main theorem below.

2.5 EXTENSION OF MODELS AND PRESENTATION OF THEORIES.

(i) $\operatorname{Each} M \in \operatorname{Mod}(\Sigma, \underline{B})$ extends uniquely to $\bar{M} \in F u n c t,(F \Sigma, \underline{B}), F \Sigma \in T h I$ being the free theory generated by $\Sigma \in G^{2}$ aph $_{I^{*}}$. This defines an isomorphism $\operatorname{Mod}(\Sigma, \underline{B}) \cong \operatorname{Funct}_{X}(F \Sigma, \underline{B})$ of categories compatible with the forgetful functors

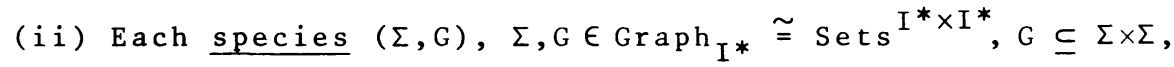
presents the theory $T=F \Sigma / \bar{G}$, and Funct $(T, \underline{B})$ is isomorphic 
to $\operatorname{Mod}((\Sigma, G), \underline{B})$ the full subcategory of $\operatorname{Mod}(\Sigma, \underline{B})$ consisting of those $M: \Sigma \rightarrow \underline{B}$ satisfying G, i.e. $\bar{M}: F \Sigma \rightarrow \underline{B}$ equalizes $\underset{\mathrm{Gr}}{\stackrel{\mathrm{pr}}{\stackrel{\mathrm{pr}_{2}}{\longrightarrow}} \mathrm{F} \Sigma}$

(iii) Each $T \in T h$ has an algebraic presentation $(\Sigma, G)$, i.e. $\Sigma, G \in \operatorname{Sets}^{*} \times I$.

PROOF. (i): M admits a full image factorization $\Sigma \stackrel{M_{0}}{\longrightarrow} T_{M} \stackrel{M^{\circ}}{\longrightarrow}$ with $T_{M} \in T_{I}$ defined by $T_{M}(A, B)=\underline{B}(M A, M B)$ with composition, identities, and projections inherited from $\underline{B}$. The Graph $I^{*}$-morphism $M_{0}$ extends uniquely to $\bar{M}_{0}: F \Sigma \rightarrow T_{M}$ in Th ${ }_{I}$ by the theorem. $\bar{M}=M^{0} \bullet \bar{M}_{0}$ is then the unique extension of M. The statement now follows from the observation that homomorphisms $f: M \rightarrow M^{\prime}$ are just models in $\underline{B}^{2}=\underline{B}^{0 \rightarrow 1}$ which followed by the evaluations $\underline{B} \underline{2} \rightarrow \underline{B}$ at 0 and 1 give $M$ and $M^{\prime}$. This yields a bijection between the Hom-sets, compatible with the forgetful functors, hence functorial.

(ii) follows from 1.5 and $F \Sigma / \bar{G}$ being a coequalizer of $\mathrm{pr}_{1}, \mathrm{pr}_{2}: G=F \Sigma$ relative $V$; for the homomorphisms cf. proof of (i). (iii) follows from the fact that each $T \in T h_{1}$ is generated by its arrows with codomain in $I$, since any arrow is induced by those.

2.6 THEOREM. For an I-sorted algebraic theory $\mathrm{T} \in \mathrm{Th}_{\mathrm{I}}$ [finitely presented] and [ $\underline{B}$ an elementary topos with NNo or] $\underline{B}$ a wel1-powered cartesian closed category with coequalizer-mono factorization and coequalizers closed under composition, the category Funct ${ }_{X}(T, \underline{B})$ of $T$-models in $\underline{B}$ has an algebraic forgetful functor U: Funct $(T, \underline{B}) \rightarrow \underline{B}^{I}$ of finite rank.

PROOF. By 2.5, (ii) and (iii) it is sufficient to show that $\operatorname{Mod}((\Sigma, G), \underline{B}) \stackrel{f u l 1}{\longrightarrow} \operatorname{Mod}(\Sigma, \underline{B}) \rightarrow \underline{B}^{I}$ is algebraic of finite rank 
for $\Sigma, G \in \operatorname{Sets}^{*} \times I, G \subseteq F \Sigma \times F \Sigma$. The second factor has this property by theorem 2.2, [for $\underline{B}$ being an elementary topos with NNo the existence of a left adjoint is guaranteed by J o h n t o n e's theorem]. Fulfillment of equations $A \stackrel{(u, v)}{\longrightarrow}$ i in G by $\Sigma$-models is clearly stable under subobjects and products and also under quotients and filtered colimits, since $x$ simultaneously preserves regular epis and commutes with filtered colimits. This implies the theorem by Lifting theorem, Birkhoff-inclusion theorem and composition theorem.

By the triangle-theorem we get immediately:

2.7 COROLLARY. For $\underline{B}$ as above and a morphism $t: T^{\prime} \rightarrow T$ in $T h{ }_{I}$ the "algebraic" functor Funct $\operatorname{Fu}_{x}(t, \underline{B}): \operatorname{Funct}_{x}(T, \underline{B}) \rightarrow_{\text {Funct }_{X}}\left(\mathrm{~T}^{\prime}, \underline{B}\right)$ is algebraic of finite rank.

2.8 EXAMPLES. The conditions for $\underline{B}$ are satisfied by Sets, Sets $\underline{C}$ ( C sma11, "presheaves"), Grothendieck-topoi(full subcategories of sets $\underline{C}$ of $j$-sheaves for a topology $j$ on $\underline{C}$ ), $\operatorname{Graph}(\cong \operatorname{sets} \stackrel{\bullet}{=}), \operatorname{Graph}_{M}\left(\cong \operatorname{Sets}{ }^{M \times M}\right), \operatorname{Cat}(\operatorname{cf} \cdot[16] 3.5)$, CGHaus (compactly generated Hausdorff spaces (cf. [18])), Tol(tolerance spaces (cf. [17]:4.9,7)). An analysis of the proofs shows that the theorem is still valid for countable $T$ (i.e. generated by a countable $\Sigma$ ) and the category of countable sets, since $B \times-$ still preserves colimits although it has no right adjoint.

A long 1 ist of 1 -sorted algebraic theories (presented by operations and equations) is given in [3]:3.

Examples for the many-sorted case: 
1. LEFT ACTIONS (called monoid automata in [17]: 5.6):

$\Sigma: \quad 1 \stackrel{1}{\longrightarrow} \mathrm{R} \stackrel{\cdot}{\mathrm{R}} \times \mathrm{R}$

$\mathrm{R} \times \mathrm{A} \stackrel{*}{\longrightarrow} \mathrm{A}$

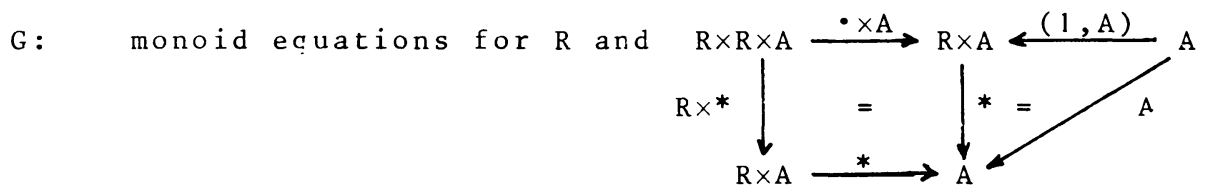

models: Lact( $\underline{B}$ ) (left actions in $\underline{B}$ (with varying scalar monoid $R$ )).

1. MOdulEs: $\Sigma$ as in 2.1. G(unitary ring equations for $R$, abelian group equations for $A$, and the above equations for *). Similarly: R, S-bimodules and group operations for $R$ a group.

3. AUTOMATA:

$\begin{array}{lll}\text { Medvedev-automata } & \Sigma: & \mathrm{I} \times \mathrm{S} \stackrel{\delta}{\longrightarrow} \mathrm{S} \\ \text { Mealy-automata } & \Sigma: & 0 \stackrel{\lambda}{\longleftarrow} \mathrm{I} \times \mathrm{S} \stackrel{\delta}{\longrightarrow} \mathrm{S} \\ \text { Moore-automata } & \Sigma: & \mathrm{I} \times \mathrm{S} \stackrel{\delta}{\longrightarrow} \mathrm{S} \stackrel{\mu}{\longrightarrow} \mathrm{O}, \text { no equations. }\end{array}$

2.9 REMARK: The conclusion in 2.6 remains valid for many other categories, too, if we replace "algebraic" by "monadic". Especially this holds for every category $\underline{B}$ with an INS-functor $\mathrm{V}: \underline{B} \longrightarrow$ Sets in the sense of [19] or equivalently $\underline{B}$ being a Topcategory in the sense of [20]. In this case $U: \operatorname{Mod}(\Sigma, \underline{B}) \longrightarrow \underline{B} I$ creates absolute coequalizers, and we have the following commutative diagram of functors:

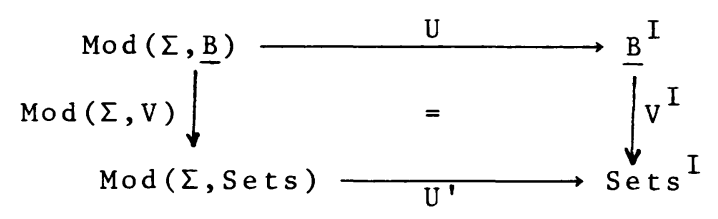

$U^{\prime}$ has a left adjoint by 2.6 and $\operatorname{Mod}(\Sigma, V)$ has a left adjoint, too (take the "discrete structure" on every component of a model in Sets yielding a model in $\underline{B}$ ). 
$\mathrm{V}^{\mathrm{I}}$ is an INS-functor and has also a left adjoint ("discrete structure"), hence by the triangle-theorem ([9]) U has a left adjoint, too. It follows from the $P$ a $r$ - criterion that $U$ is monadic. This holds e.g. for $\underline{B}$ being the category of topological, uniform, measurable, compactly generated, limit spaces (cf.[19] for other examples.

Note that the induced functor $\operatorname{Mod}(\Sigma, V): \operatorname{Mod}(\Sigma, \underline{B}) \rightarrow \operatorname{Mod}(\Sigma, \operatorname{Sets})$ is again an INS-functor. The initial object can be defined in each component of the model, and it follows directly that this defines a model in $\underline{B}$. In the one-sorted case this is mentioned in [19]. Hence $\operatorname{Mod}(\Sigma, \underline{B})$ is complete and cocomplete (cf. [19]: 1.6) which will be used later (cf.3.4).

\section{ALGEBRAS WITH ACTIONS}

The examples at the end of section 2 become more interesting for the applications, if the "scalar" or "input" components are fixed, cf. [17] and the examples in 3.4 and 3.5 . We show here that under certain conditions (3.1, 3.2) fixing components still gives monadic or algebraic forgetful functors.

\section{1 ALgEBRAS WITH ACTIONS: A theory with J-action ( $\in$ Sets)} is an I $\dot{U} \mathrm{~J}$-sorted theory $\mathrm{T}$ having the following factorization property:

Every $T$-arrow $A \times B \stackrel{w}{\longrightarrow} A^{\prime}\left(A, A^{\prime} \in J^{*}, B \in I^{*}\right)$ has a factorization $\mathrm{A} \cong \mathrm{B} \stackrel{\mathrm{pr}}{\longrightarrow} \mathrm{A} \stackrel{\hat{w}}{\longrightarrow} \mathrm{A}^{\prime}$.

Now let $T_{J} \in T_{J}$ be the full subcategory of $T$ with object set J* (Example: $T_{J}=$ theory of unitary $r i n g s$ ) and $R \in \operatorname{Mod}\left(T_{J}, \underline{B}\right)$ be a fixed $\mathrm{T}_{\mathrm{J}}$-model over some base category $\underline{B}$ with (given) finite products. Then a T-model M with $\mathrm{M}_{\mathrm{T}_{\mathrm{J}}}=\mathrm{R}$ is called an R-model (for $\mathrm{T}$ ). Together with homomorphisms $f=(f i)_{i \in I}{ }_{j}: M^{\prime} M^{\prime}$ with $f j=i d_{M j}$ 
for $j \in J$, these form a subcategory $\operatorname{Mod}_{R}(T, \underline{B})$ of $\operatorname{Mod}(T, \underline{B})$ with forgetful functor $U_{R}: \operatorname{Mod}_{R}(T, \underline{B}) \longrightarrow \underline{B}^{I}$. Now we give a sufficient condition for the factorization property, which is trivially satisfied in all our examples.

3.2 LEMMA: If $T \in T_{\text {IUJ }}$ is generated by $J$-actions, i.e. generated by arrows $A \longrightarrow i(i \in I \dot{U} J)$ with $A \in J^{*}$ for $i \in J$, then $\mathrm{T}$ has the factorization property of 3.1 .

PROOF: By hypothesis the arrows of a generating graph $\Sigma \in G r a p h(I \dot{U} J)^{*}$ of $T$ have the factorization property. We have to show that it is preserved under inducing morphisms into a product and under composition. The former is trivial.

Now let $\mathrm{A} \times \mathrm{B} \stackrel{\omega}{\longrightarrow} \mathrm{A}^{\prime} \times \mathrm{B}^{\prime}-\stackrel{\omega^{\prime}}{\longrightarrow} \mathrm{A}^{\prime \prime} \times \mathrm{B}^{\prime \prime}$ be in $\mathrm{T}$ with $\mathrm{A} \times \mathrm{B} \stackrel{\omega}{\longrightarrow} \mathrm{A}^{\prime} \times \mathrm{B}^{\prime} \stackrel{\mathrm{pr}}{\longrightarrow} \mathrm{A}^{\prime}=\mathrm{A} \times \mathrm{B} \stackrel{\mathrm{pr}}{\longrightarrow} \mathrm{A} \stackrel{\hat{\omega}}{\longrightarrow} \mathrm{A}^{\prime}$ and similarly for $\omega^{\prime}$. Then

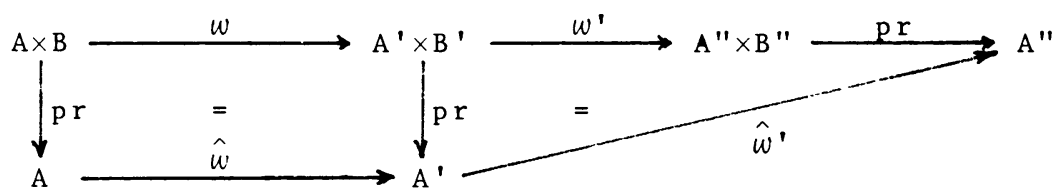

i.e. the J-part of $w^{\prime} \circ \omega$ again factorizes through $A \times B \stackrel{p r}{\longrightarrow} A . q . e . d$.

3.3 THEOREM: Let $\underline{B}$ be a category with given finite products such that for any $\mathrm{T} \in \mathrm{Th} \mathrm{I}_{\mathrm{I}}$, I $\in$ Sets arbitrary, Mod(T, $\left.\underline{B}\right)$ has coequalizers and $U: \operatorname{Mod}(\mathrm{T}, \underline{B}) \longrightarrow B^{I}$ is monadic or algebraic [of finite rank] (See 2.6 for sufficient conditions for B ). Then $U_{R}: \operatorname{Mod}_{R}(T, \underline{B}) \longrightarrow \underline{B}^{I}$ has the same property for any theory $T \in T_{I} \dot{U}$ with J-action (see 3.1 ).

PROOF: Construction of a left adjoint $\mathrm{F}_{\mathrm{R}}$ for $\mathrm{U}_{\mathrm{R}}$ We will construct $F_{R} \longrightarrow U_{R}$ using $F \longrightarrow U: \operatorname{Mod}(T, \underline{B}) \longrightarrow \underline{B}^{I} \dot{U} \mathrm{~J}$, 
$\mathrm{F}_{\mathrm{J}} \longrightarrow \mathrm{U}_{\mathrm{J}}: \operatorname{Mod}\left(\mathrm{T}_{\mathrm{J}}, \underline{\mathrm{B}}\right) \longrightarrow \underline{\mathrm{B}}^{\mathrm{J}}$ and $\mathrm{F}_{\mathrm{I}} \longrightarrow \mathrm{U}_{\mathrm{I}}: \operatorname{Mod}\left(\mathrm{T}_{\mathrm{I}}, \underline{\mathrm{B}}\right) \longrightarrow \underline{\mathrm{B}}^{\mathrm{I}}$.

Let $B=(B i)_{i \in I}$ be a given family of B-objects. For the family

$(R j, B i)_{i \in I,} \in J$ there exists the free $T-a \operatorname{lgebra} F\left((R j, B i)_{i \in I, j \in J}\right)$,

or $F(R j, B i)$ for short.

We first prove the following

LEMMA : $\quad F(R j, B i)_{\left.\right|_{T}}=F_{J} U_{J} R$.

PROOF: We show that $F(R j, B i)_{\mid J}=F(R j, B i)_{\mid T}$ solves the universal problem corresponding to $U_{J}$ for the object $U_{J} R=(R j)_{j} \in J$. Let $R^{\prime}$ be a $T_{J}$-algebra and $f=\left(f j: R j \rightarrow R^{\prime} j\right)_{j \in J}: U_{J} R \rightarrow U_{J} R^{\prime}$

a family of $\underline{B}$-morphisms. Define an extension $\bar{R}^{\prime} \in \operatorname{Mod}(T, \underline{B})$ of $R^{\prime}$ uniquely by $\bar{R}^{\prime} i=1$ (terminal object) for all $i \in I$ and $\bar{R}^{\prime}(\mathrm{A} \times \mathrm{B} \stackrel{\omega}{\longrightarrow} \mathrm{A})=\mathrm{R}^{\prime}(\hat{\omega}) \bullet \mathrm{pr}$ for $\mathrm{A} \in \mathrm{J}^{*}$ where $\hat{\omega}$ is given by the factorization property 3.1 .

So by our assumptions there exists a unique T-homomorphism $\overline{\mathrm{f}}: F(\mathrm{Rj}, \mathrm{Bi}) \rightarrow \overline{\mathrm{R}}^{\prime}$ such that diagram (1) commutes:

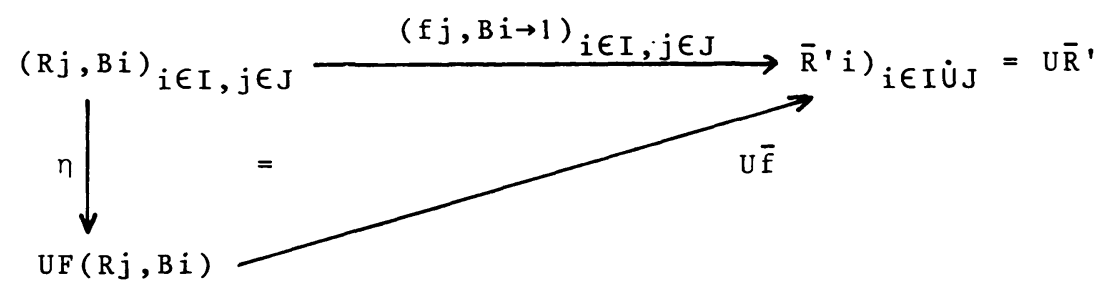

The lemma follows now from $\bar{R}^{\prime} I_{J}=R^{\prime}$ and by considering the $J-$ components of (1) (existence of an extension of ( $f j)_{j \in J}$ ) and by the observation that any $\overline{\mathrm{f}}: \mathrm{F}(\mathrm{Rj}, \mathrm{Bi})_{\mid \mathrm{T}} \longrightarrow \mathrm{J}^{\prime}$ in $\operatorname{Mod}\left(\mathrm{T}_{\mathrm{J}}, \underline{B}\right)$ extends to an $\overline{\mathrm{f}}: \mathrm{F}(\mathrm{Rj}, \mathrm{Bi}) \longrightarrow \overline{\mathrm{R}}^{\prime}$ in $\operatorname{Mod}(\mathrm{T}, \underline{B})$ (uniqueness of the extension). q.e.d. 
Now let $\varepsilon$ be the back-adjunction for $F_{J} \rightarrow U_{J}$, i.e.

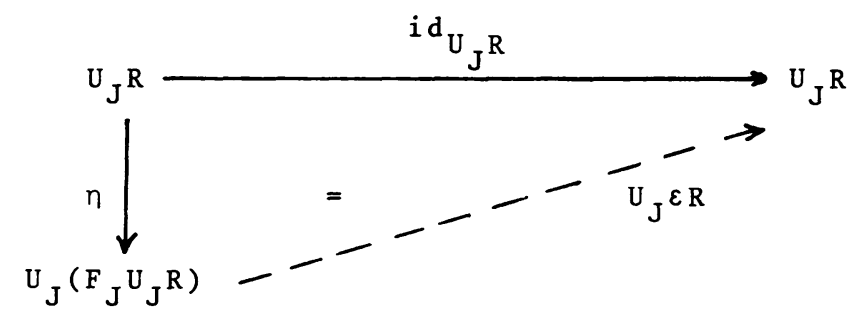

and $P \frac{P_{1}}{P_{2}} F_{J} U_{J} R$ the kernel pair of $\varepsilon R$ in $\operatorname{Mod}\left(T_{J}, \underline{B}\right)$.

Define the $\underline{B}^{I \dot{U} J}$-morphism pair $\left(\mathrm{r}_{1}, \mathrm{r}_{2}\right)$ by

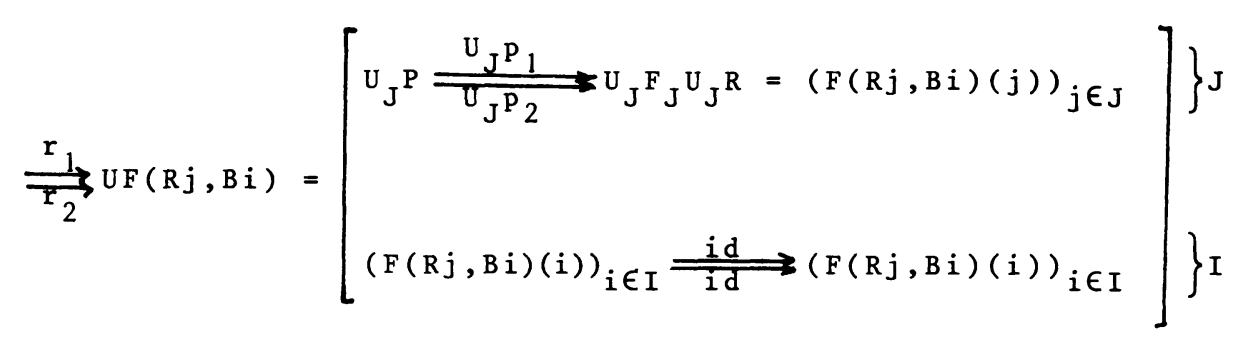

Define $F_{R}\left((B i)_{i \in I}\right)=\operatorname{Coeq}\left(r_{1}, r_{2}\right)$ to be the coequalizer of $\left(r_{1}, r_{2}\right)$ relative $U$ in $\operatorname{Mod}(T, \underline{B})$, and the front adjunction

$\tilde{n}:(B i)_{i \in I} \longrightarrow U_{R} F_{R}\left((B i)_{i \in I}\right)$ as the composition in

( 2

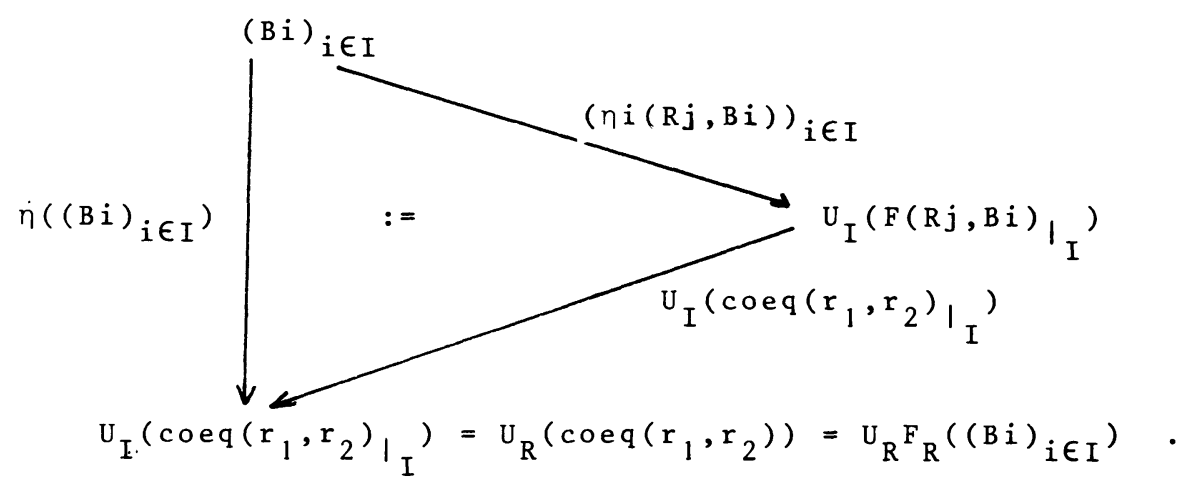

It remains to prove

(i) $\operatorname{Coeq}\left(\mathrm{r}_{1}, \mathrm{r}_{2}\right) \in \operatorname{Mod}_{\mathrm{R}}(\mathrm{T}, \underline{B})$

(ii) the universal property of a free construction with respect the forgetful functor $U_{R}$. 
(i) We have to verify $\operatorname{Coeq}\left(r_{1}, r_{2}\right)_{I_{J}}=R$.

We will show that $\operatorname{coeq}\left(\mathrm{r}_{1}, \mathrm{r}_{2}\right)_{\mathrm{J}_{\mathrm{J}}}$ is a coequalizer of $\left(\mathrm{p}_{1}, \mathrm{p}_{2}\right)$ in $\operatorname{Mod}\left(\mathrm{T}_{\mathrm{J}}, \underline{B}\right)$. Let $\mathrm{g}: \mathrm{F}_{\mathrm{J}} \mathrm{U}_{\mathrm{J}} \mathrm{R} \longrightarrow \mathrm{M}$ be a $\mathrm{T}_{\mathrm{J}}$-homomorphism satisfying $\mathrm{g} \bullet \mathrm{p}_{1}=\mathrm{g} \bullet \mathrm{p}_{2}$. By an argument used in the lemma, $M$ can be extended in a unique way to a $T$-model $\bar{M}$ by $\bar{M} i=1$ for all $i \in I$, and $g$ can be extended uniquely to a T-homomorphism $\overline{\mathrm{g}}: \mathrm{F}(\mathrm{Rj}, \mathrm{Bi}) \longrightarrow \overline{\mathrm{M}}$. It follows that $U \bar{g} \bullet r_{1}=U \bar{g} \circ r_{2}$ and hence by definition of the coequalizer Coeq $\left(r_{1}, r_{2}\right)$ relative $U$ there exists a unique T-homomorphism $h: \operatorname{coeq}\left(r_{1}, r_{2}\right) \longrightarrow \bar{M}$ satisfying $h$ ocoeq $\left(r_{1}, r_{2}\right)=g$, and the I-components of $h$ are the terminal morphisms into 1 , of course, which implies that the restriction $h_{/ J}$ is the unique $T_{J}$-homommorphism satisfying $h_{I_{J}} \circ \operatorname{coeq}\left(r_{1}, r_{2}\right)_{I_{J}}=\bar{g}_{I_{J}}=g$. This implies that Coeq $\left(r_{1}, r_{2}\right){ }_{J}$ is a coequalizer of $\left(p_{1}, p_{2}\right)$ and hence isomorphic to $R$ because $\left(p_{1}, p_{2}\right)$ was the kernel pair of the coequalizer $\varepsilon R$. By changing the representant of $\operatorname{coeq}\left(r_{1}, r_{2}\right)$ we get equality. (More precisely: use the fact that $U: \operatorname{Mod}(T, \underline{B}) \longrightarrow \underline{B}^{I \dot{U} J}$ creates isomorphisms).

(ii) Let $\tilde{M}: T \longrightarrow \underline{B}$ be an $R$-model and $f=(f i: B i \rightarrow \widetilde{M} i)_{i \in I}$ a family of $\underline{B}$-morphisms. With $f_{j}=i_{R j}$ for each $j \in J$ there exists a unique T-homomorphism $\overline{\mathrm{f}}: \mathrm{F}(\mathrm{Rj}, \mathrm{Bi}) \longrightarrow \widetilde{\mathrm{M}}$ in $\operatorname{Mod}(\mathrm{T}, \underline{B})$ satisfying

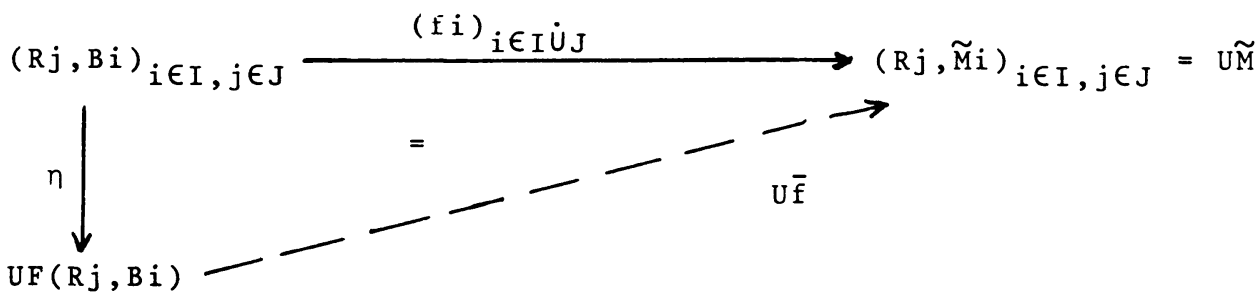

Considering only the J-components we get the equation

$$
\overline{\mathrm{f}}_{\mathrm{J}_{\mathrm{J}}}=\varepsilon \mathrm{R}
$$




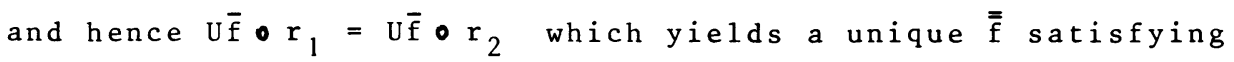

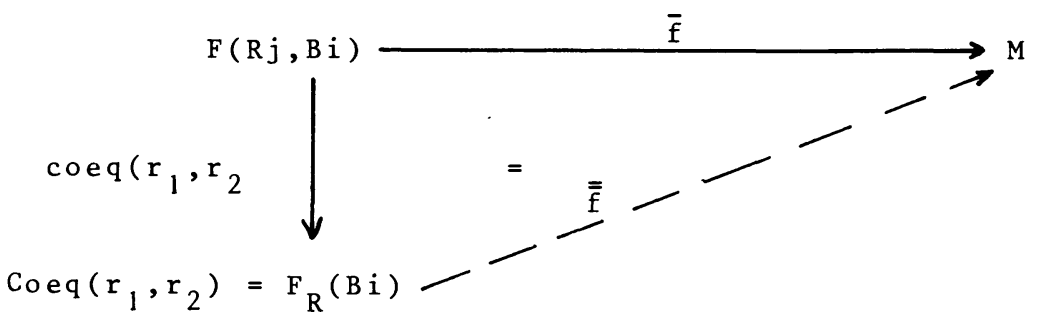

which in fact is a $\operatorname{Mod}_{R}(T, \underline{B})$-morphism because coeq $\left(r_{1}, r_{2}\right)_{J_{J}}=$ $=\varepsilon R=\overline{\mathrm{f}}_{\mathrm{J}}$.

The uniqueness of $\overline{\bar{f}}$ follows stepwise.

This proves $U_{R}$ to have a left adjoint $F_{R}$.

$U_{R}$ creates absolute coequalizers, since $U$ does and identities can

be taken as the J-components of this coequalizer. Hence $U_{R}$ is monadic by the $P$ a $r$ é-criterion 1.1. The same argument shows that $U_{R}$ creates coequalizers of $\left(U_{R^{-}}\right)$kernel pairs [and filtered colimits] if U does. q.e.d.

3.4 APPLICATIONS AND EXAMPLES: For applying theorem 3.3 the conditions concerning the base category $\underline{B}$ are satisfied by all categories mentioned in 2.8 and 2.9: Sets, Sets , Countable sets, Graph, Cat, CGHaus, Tol and all Top-Categories over Set, e.g. Top, CG, Unif, Meas, Lim...

Furthermore the factorization property of the theory $T$ (cf.3.1, 3.2) is satisfied in all the examples in 2.8 , if we fix the monoid, the ring, or the input object as needed.

Left R-actions and (left) $\underline{\text { R-modules }}$ over $\underline{B}$ for a fixed R:

of particular interest are topological R-modules for a topological unitary ring $R$ and $\underline{R \text {-modules }}$ for a ring-object $R$ in a category $\mathrm{Sh}_{\mathrm{j}}(\underline{C})$ of set valued sheaves, i.e. $R$ a sheaf of rings. By the theorem 3.3, this category has an algebraic forgetful functor $\mathrm{U}: \operatorname{Mod}_{R} \longrightarrow \mathrm{Sh}_{\mathrm{j}}(\underline{\mathrm{C}})$. By the triangle theorem we get an algebraic 
forgetful functor $\operatorname{Mod}_{R} \longrightarrow A b$ into the category of abelian group objects in $\mathrm{Sh}_{\mathrm{j}}(\underline{\mathrm{C}})$, i.e. into the category of sheaves with values in abelian groups. The same is true for $R-S-b i m o d u l e s$ in $S_{j}(C)$. Automata with fixed input I: Corresponding to the three types of automata mentioned in $2.8 \operatorname{Mod}_{I}(T, \underline{B})$ for a fixed $I \in \underline{B}$ becomes the category of Medvedev-, Mealy- or Moore-automata with fixed input respectively (the category of Medvedev-automata with fixed input is denoted by $\underline{B}-\underline{\text { Medv }}$ in [17]).

In each case the forgetful functor to $\underline{B}$ resp. $\underline{B}^{2}$ is algebraic or monadic depending on the base category (cf. 2.9).

of special interest are deterministic, topological, compactly generated, and tolerance automata (cf.[17], [21]). Automata over $\underline{B}=$ Cat are useful for the theory of forma1 languages (cf.[22]). By algebraicity or monadicity of the forgetful functor there follow most of the structural properties of automata mentioned in [17] sect. 11 .

Note that in case of fixed output 0 the forgetful functor is not monadic, in fact the factorization property is not fulfilled.

3. 5 GENERALIZATION TO MONOIDAL CATEGORIES. The results of section 2 are preserved, if the I-sorted $L$ a $v$ e $r$ theories are replaced by [symmetric] monoidal theories and $\underline{B}$ in theorem 2.6 is monoidal closed instead of cartesian closed (and 0 preserves simultaneously coequalizers of kernel pairs), see [15], and [16] for the heterogeneous case. The same is true for theorem 3.3 because of its "relative" formulation and since we did not use the universal properties of $x$ in the proof.

A list of [symmetric] monoidal categories which are "nice" in the above sense is to be found in [15]:3.11. Theorem 3.3 then gives in particular the following examples of action-categories with 
algebraic forgetful functors: Left action over abelian groups

( $\underline{R-\operatorname{modul} e s)}, \underline{\text { partial }}$ and bilinear automata, and topological automata with $\delta, \lambda$ continuous in each component separately (using $($ Top,,$))(\operatorname{cf} .[17],[21])$.

\section{REFERENCES}

1. SEMINAR on Triples and Categorical Homology Theory, BerlinHeidelberg-New York 1969 (L.N. in Math. 80).

2. Maclane, S. Categories for the Working Mathematician, New York-Heidelberg-Berlin 1972 .

3. PAREIGIS, B. Categories and Functors, New York-London 1970.

4. PFENDER, M. Kongruenzen, Konstruktion von Limiten und Cokernen und algebraische Kategorien, Dissertation TU Berlin 1971 .

5. MAHR, B. Erzeugung von Kongruenzen, Diplom Arbeit TU Berlin 1974.

6. HANSEN, H. Kategorielle Betrachtungen zu den Sätzen von Jordan-Hölder und Schreier, Diplom Arbeit TU Berlin 1974.

7. EHRIG, H./PFENDER, M. u.a. Kategorien und Automaten, BerlinNew York 1972 .

8. LINTON, F.E.J. Coequalizers in Categories of Algebras, in [ 1 ], 75-90.

9. DUBUC, E.J. Adjoint Triangles, in: Reports of the Midwest Category Seminar II, Berlin-Heidelberg-New York 1968, 69-91 (L.N. in Math.61).

10. HERRLICH, H. Topologische Reflexionen und Coreflexionen, Berlin-Heidelberg-New York 1968 (L.N. in Math. 78).

11. LAWVERE, F.W. Functorial Semantics of Algebraic Theories, Ph.D. -Thesis, Columbia-University 1973, summarized in: Proc. Nat. Acad. Sci. USA 50 (1963), 869-873.

12. HIgGins, P.J. Algebras with a Scheme of Operators, Math. Nachr. 27 (1963), 115-132.

13. BIRKHOFF, G./LIPSON, J.D. Heterogeneous Algebras, J. Comb. Theory 8 (1970), 115-133.

14. SCHULTE-MÖNTING, J. Kategorien von Algebren mit einer Familie von Grundobjekten, Diplom Arbeit Universität Freiburg/Br. 1968 .

15. PFENDER, M. Universal Algebra in S-tanoidal Categories, Algebra-Berichte Math. Inst., Univ. München 20 (1974).

16. PFENDER, M. Universelle Algebren in monoidalen Kategorien, Manuskript TU Berlin 1972 . 
17. EHRIG, H./KIERMEIER, K.-D./KREOWSKI, H. - J . /KÜHNEL, W. Universal Theory of Automata, Stuttgart 1974.

18. STEENROD, N.E. A Convenient Category of Topological Spaces, Mich. Math.J. 14 (1967), 133-152.

19. WISCHNEWSKY, M. Partielle Algebren in Initialkategorien, Math. Z. 127 (1972), 83-91.

20. WYLER, 0. Categories of General Topology, Arch Math. 22 (1971), 7-17.

21. EHRIG, H. / KÜHNEL, W. Topological Automata, Revue Franc. $\frac{\text { d'Automatique, Informatique et Recherche Opérationnelie }}{8 \text { R-3(1974), 73-91. }}$

22. BENSON, D.B. An Abstract Machine Theory for Formal Language Parsers, in:

Category Theory Applied to Computation and Control, Proc eedings of the First International Symposium San Francisco $1974,106-111$, Berlin - HeidelbergNew York 1975 (Lectures Notes in Computer Science 25)

23. KÜHNel, W. / MESEguer, J. / PFEnder, M. / SOls, I. Primitive Recursive Algebraic Theories and Program Schemes, Bul1. Austr. Math. Soc. 17 (1977), $207-233$

24. FREYD, P. Aspects of Topoi, Bul1. Austr. Math. Soc. $\underline{7}$ (1972), $i-76$ 


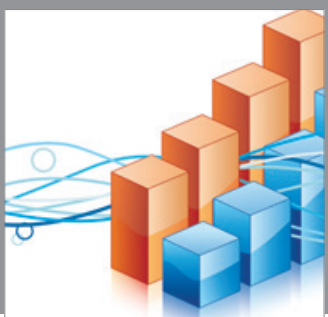

Advances in

Operations Research

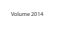

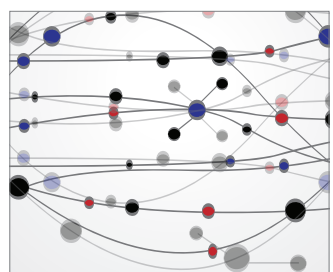

\section{The Scientific} World Journal
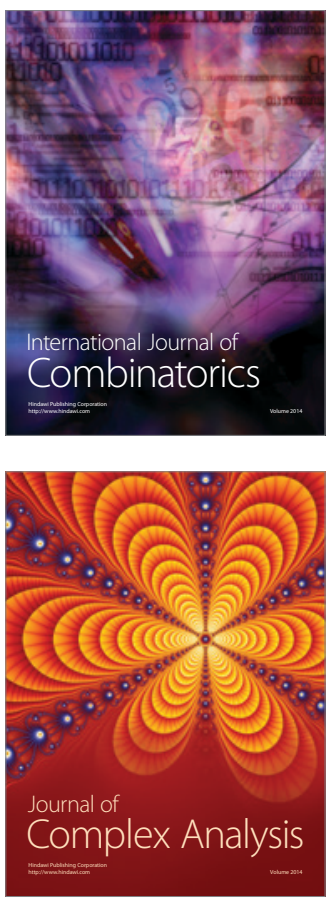

International Journal of

Mathematics and

Mathematical

Sciences
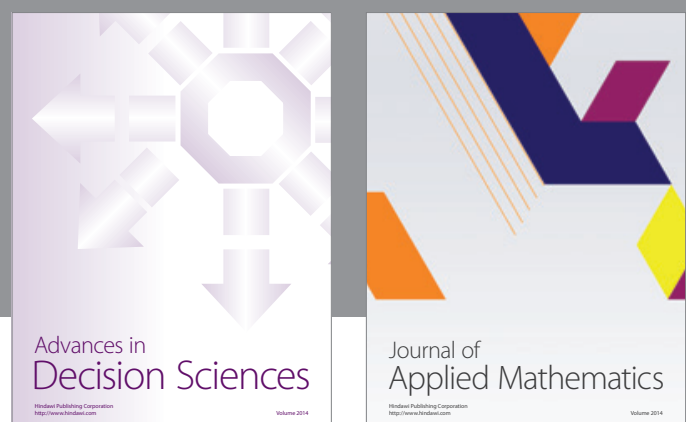

Journal of

Applied Mathematics
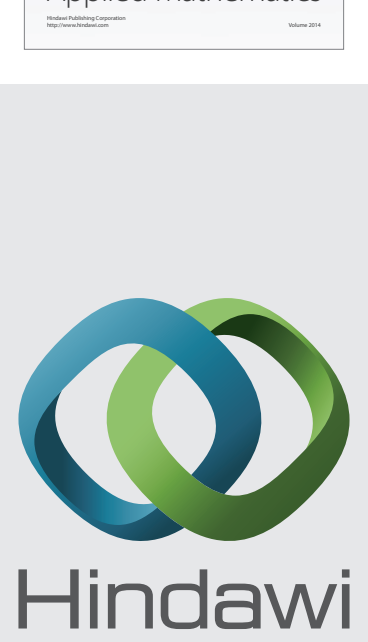

Submit your manuscripts at http://www.hindawi.com
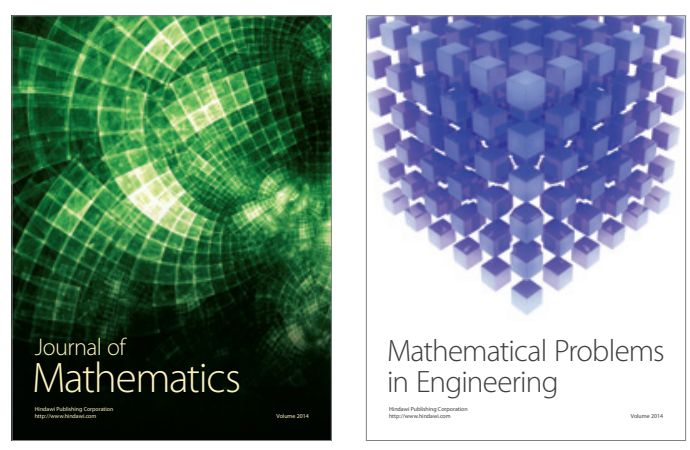

Mathematical Problems in Engineering
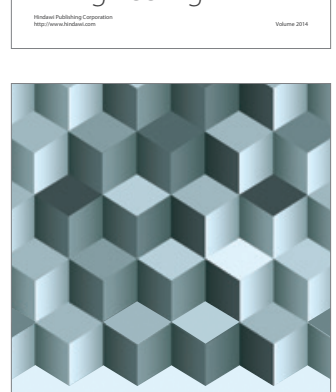

Journal of

Function Spaces
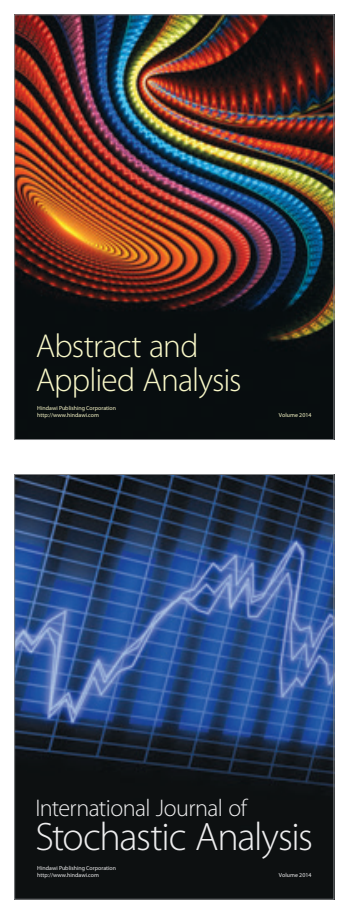

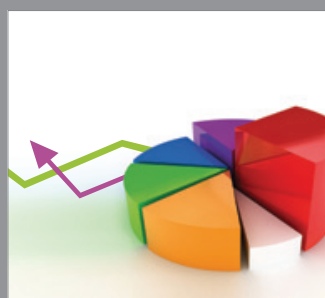

ournal of

Probability and Statistics

Promensencen
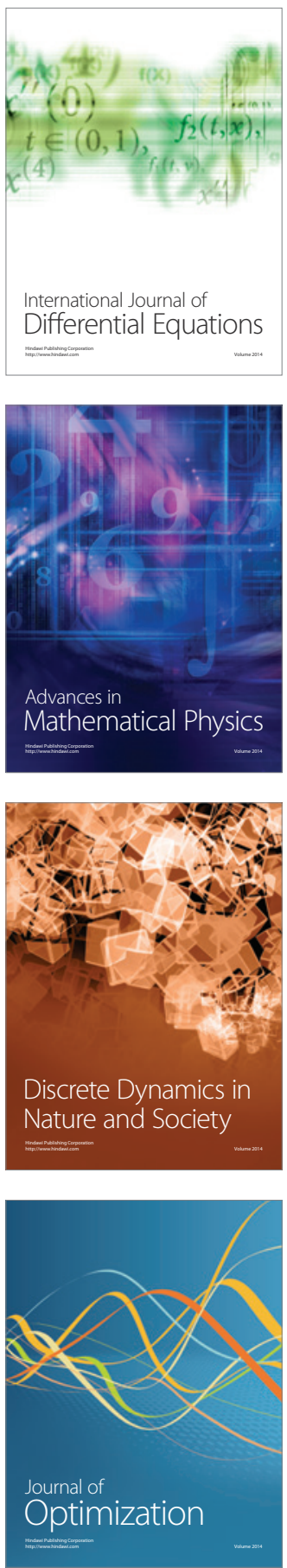